\section{Recent Mathematical Stamps: 2007, The Golden Ratio}

n 2007, as part of a series in science and technology, the Macau Post Office issued a set of stamps featuring topics related to the golden ratio, $\Phi=(1+\sqrt{ } 5) / 2$ :

- the Fibonacci sequence $1,2,3,5,8,13, \ldots$, illustrated by numbers of rabbits (from the problem that gave rise to it);

- two intersecting sets of spirals, as may be seen on a sunflower;

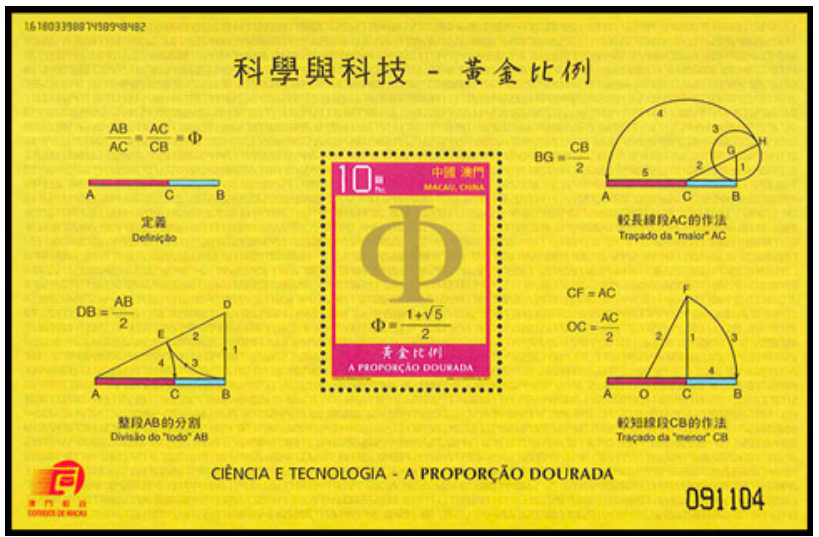

- Penrose tilings, constructed from two shapes known as kites and darts;

- the spiral pattern that appears on a nautilus shell.

There was also a souvenir sheet featuring the golden ratio and a number of constructions that lead to it.

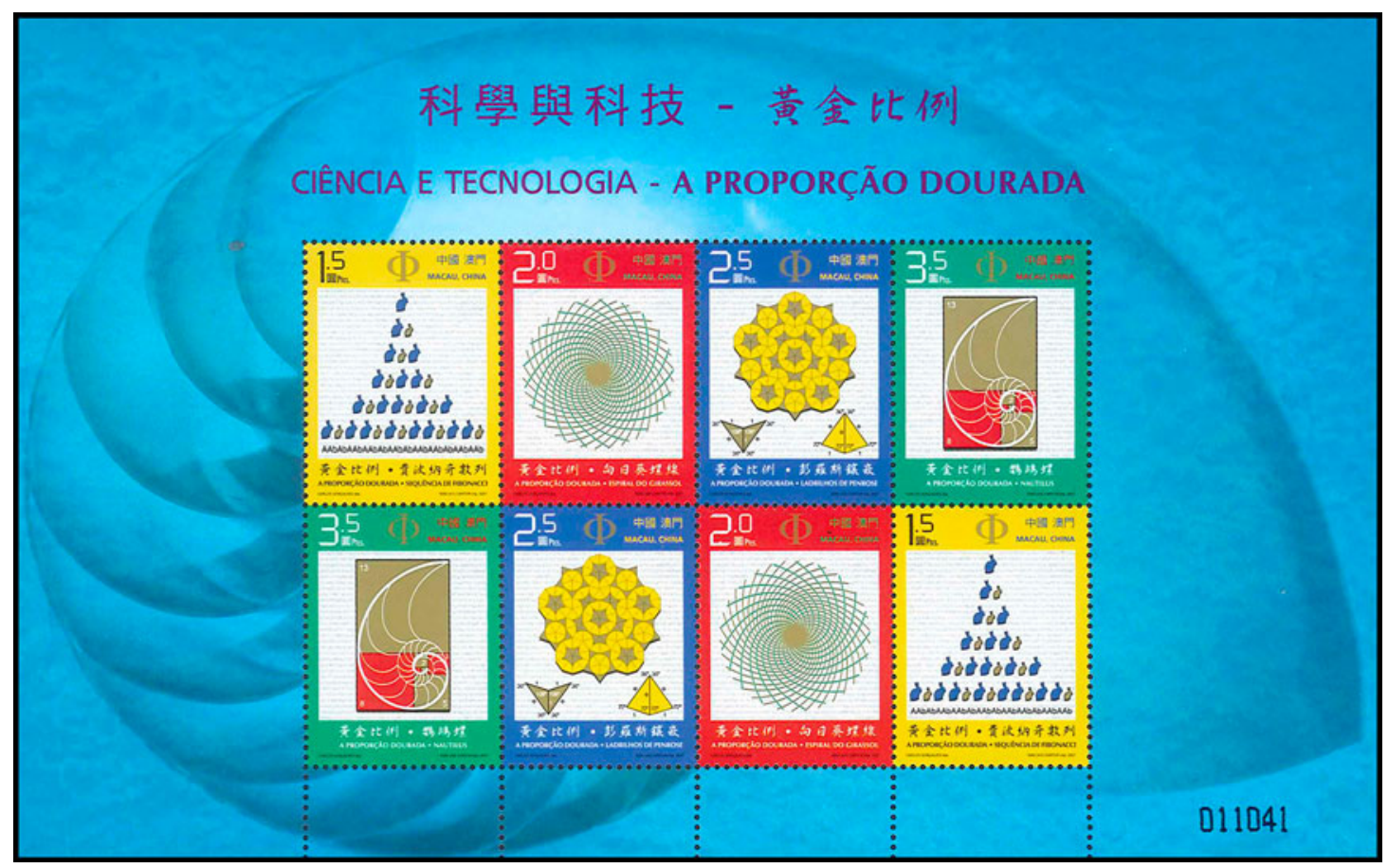

$>$ Please send all submissions to the Stamp Corner Editor,

Robin Wilson, Faculty of Mathematics,

Computing and Technology

The Open University, Milton Keynes, MK7 6AA, England

e-mail: r.j.wilson@open.ac.uk 